\title{
Videogames and Art: Comparing Emotional Feedback from Digital and Classic Masterpieces
}

\author{
Roberto Dillon ${ }^{1}$ \\ ${ }^{1}$ School of Business, James Cook University, Singapore
}

\begin{abstract}
Can Videogames be considered Art? The present study analyzes this question from an emotional perspective: in other words, if we see Art as something designed to evoke emotions through fantasy, are the emotional experiences we have while looking at works of Art similar to the ones we have while playing a video game? An experiment centered around seven highly acclaimed video games and seven art masterpieces from different periods was carried out at James Cook University to answer this question. The works have been discussed by using the Geneva Emotion Wheel as well as another set of emotional indicators, here proposed for the first time. Results have been compared and statistically analyzed to show that, indeed, Art and videogames can elicit very similar emotional experiences in people, showcasing strong similarities between the two media.
\end{abstract}

Keywords - Art, Emotions, Geneva Emotion Wheel, Singapore Engagement Map, Videogames

\section{INTRODUCTION}

Whether video games can be considered an "Art" form or not has been subject to very heated debates, especially since Kroll stated that "games [...] can't transmit the emotional complexity that is the root of art" [1]. This perspective was later reinforced in [2].

On the other hand, game designers [3] and scholars [4], [5] tried to counter such negative views by proposing a different perspective, generally expressed in qualitative terms or via discussions that are more philosophical than technical in nature. Lately, these included some very interesting analyses comparing specific games with well-established philosophical frameworks like Arthur Danto's theory of innovation in Art [6] or even comparing game designers to moral teachers by using Kant's Ethics [7].

The present study, on the other hand, tries to further the discussion by following a more experimental route leading to investigating the similarities between Art and Video Games in quantitative and measurable terms so as to debate the original Kroll's negative assertion with actual hard data.

To do so, we first need to define what "Art" is and then determine whether and how we can relate video games to eventually fit into this definition.

As stated by Kroll, emotions have always been considered a fundamental characteristic of artistic expression and this was also the focus of the original "games as art" assertion made by game designer Chris Crawford in his seminal work "The Art of Computer Game Design" [8]:

"Art is something designed to evoke emotion through fantasy. The computer game is an art form because it presents its audience with fantasy experiences that stimulate emotions"

By taking these statements as possible definitions ("Emotional complexity is at the root of Art" and "Art is something designed to evoke emotion through fantasy"), it seems logical then to compare Art and Videogames in terms of the emotions they can evoke in people and see if these show similarities or statistically meaningful differences. In other words, can universally recognized art masterpieces and critically acclaimed video games stimulate the same emotions in viewers and players alike? If so, video games could effectively be considered an "Art" form in their own right.

To answer this question an experiment was designed where different testers where asked to analyze a few well known paintings belonging to different authors and historical periods as well as play a few modern video games chosen due to their critical acclaim and often described as "artistic" by the specialized media. For each of these they would then fill an evaluation form describing their emotional experience in quantitative terms.

\section{EXPERIMENT AND METHODOLOGY}

The experiment was divided into two phases, one for the artwork and one for the games. Paintings were selected according to the following criteria: 
1. They had to showcase a different set of artists and styles from different time periods but without including works that were too abstract in nature.

2. They had to depict different environments and situations, including either calm or action scenes.

3. They had to be well known, critically acclaimed masterpieces.

In the end, among the many possibilities, it was decided to focus the study on the following seven paintings:

1. Supper at Emmaus (Michelangelo Merisi da Caravaggio, 1571-1610)

2. Lion Hunt (Peter Paul Rubens, 1577-1640)

3. Yonker Ramp and his Sweetheart (Frans Hals, 1580-1666)

4. The Nightmare (Henry Fuseli, 1741-1825)

5. The Third of May 1808 (Francisco Goya, 1746-1828)

6. Garden at Sainte-Addresse (Claude Monet, 1840-1926)

7. The Scream (Edvard Munch, 1863-1944)

For selecting the videogames, similar criteria were followed and it was decided to focus on games that were labeled as 'artistic' by the specialized press even if they were not necessarily blockbusters in term of sales and/or commercial success.

In particular, the following videogames were chosen:

1. Flower (thatgamecompany/SCEA, PS3, 2009)

2. Heavy Rain (Quantic Dreams/SCEA, PS3, 2010)

3. Amnesia: Dark Descent (Frictional Games, PC, 2010)

4. Ico (BluePoint Games/SCEA, PS3, 2011)

5. Shadow of the Colossus (BluePoint Games/SCEA, PS3, 2011)

6. Journey (thatgamecompany/SCEA, PS3, 2012)

7. Child of Eden (Q Entertainment/Ubisoft, PS3, 2012)

Fourteen people, including both undergraduate and graduate students, took part to the experiment which was arranged within the area of the Videogame Museum in James Cook University's Singapore campus, a quiet locale within the institution's library premises. Headphones were used to further isolate testers from the nearby environment and avoid possible distractions.

After a short briefing on the experiment and its modalities, each person had to select at least four paintings and four games of their choice. This was done so that testers could avoid art or games they did not appreciate or did not find interesting, though they could have analyzed all of them if they wished to do so. Each session lasted for a few hours where testers were instructed to take breaks and rest for a short while in between games and art samples so as to allow themselves to go back into a neutral emotional state and avoid later analyses to be affected by previous experiences. If needed, testers could break the overall experiment into independent sessions across different days, especially if playing a specific game took too long and fatigue would start affecting later analyses.

After each playing or art analysis session, testers had to fill a given questionnaire to discuss their emotional experience, answering the question "Try to identify how the game/artwork made you feel. Did you experience any of the following?"

The questionnaires were the same for both Art and Videogames and revolved around the "Geneva Emotion Wheel" as discussed in [9] and [10], a model spanning sixteen emotions ranging from Pride and Elation to Contempt, Hostility and Anger. Each emotion had to be evaluated following a five values scale from 0 to 4, labeled as "not at all / a little /some / yes / A LOT!".

Besides the generic Geneva Emotion Wheel, also another set of emotional indicators was used, inspired by the work discussed in [11] and [12], to get more specific information about the ability of the work under analysis to emotionally engage and capture people's attention in a videogame setting. In particular, the following questions were included:

- Feeling attracted to the work thanks to its Aesthetics/Visual qualities and style (synthetically identified in the following diagrams as "color appreciation").

- Feeling of Immersion (i.e. you feel like you are part of the action)

- Feeling a bond with the characters represented

- Feeling in danger

- Questioning the meaning/purpose of the work

For brevity's sake and in analogy with the Geneva Emotion Wheel, we shall refer to this model as the "Singapore Engagement Map". In the experiment, each of these questions had to be evaluated exactly 
like the individual emotions in the Geneva Emotion Wheel, i.e. on a scale ranging from 0 ("not at all") to 4 ("A LOT!").

\section{RESULTS}

Results for each work of art and each game, averaged across all testers, are shown across pictures 1 to 14 .
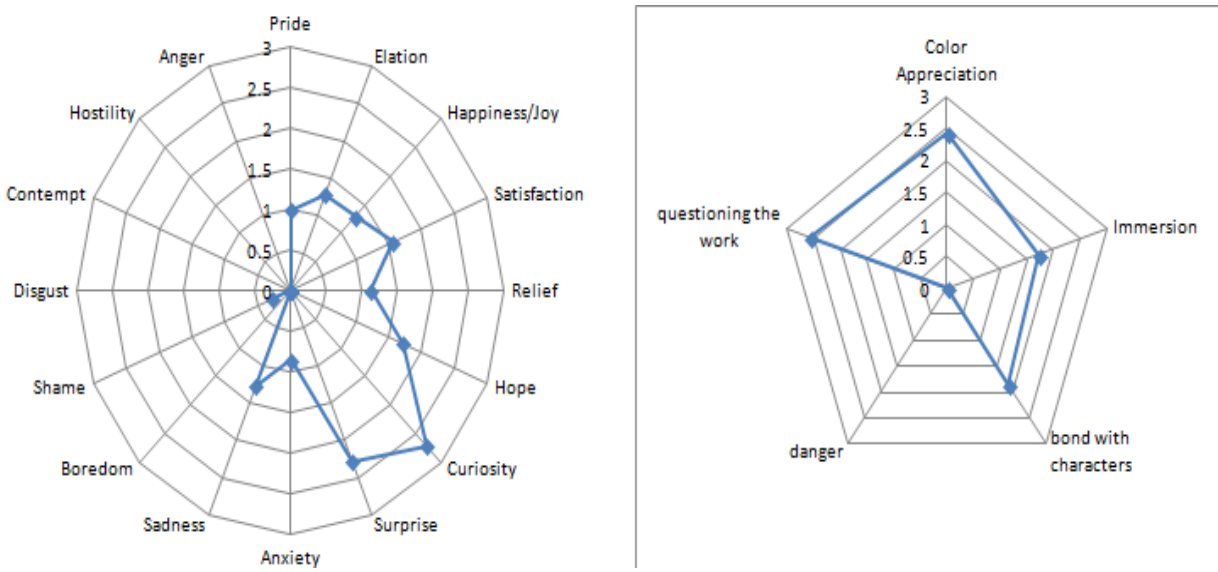

Figure 1: Supper at Emmaus: Geneva Emotion Wheel (left) and Singapore Engagement Map.
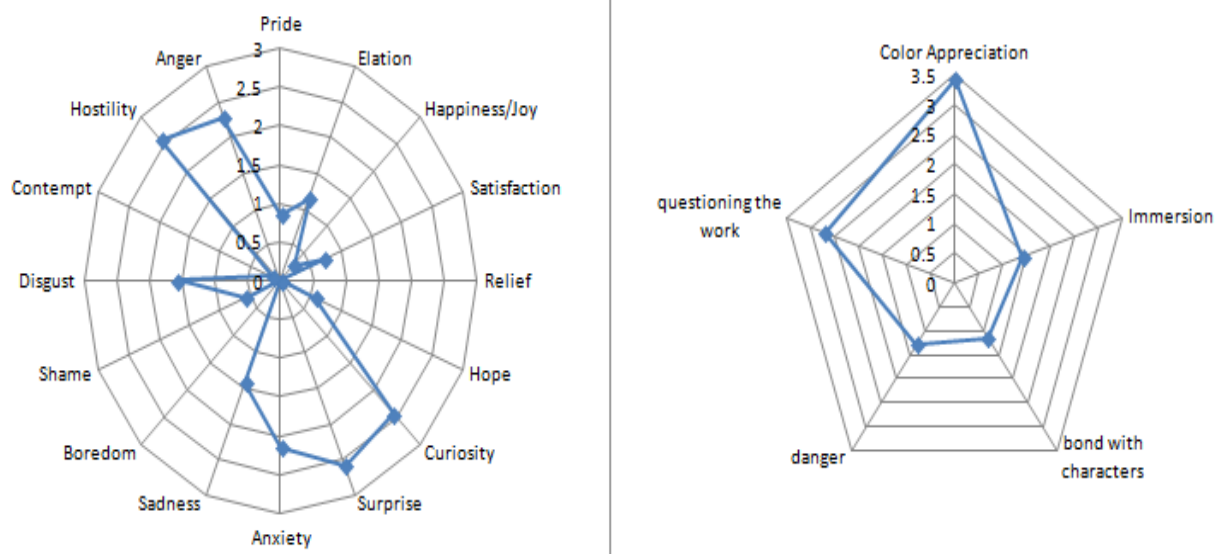

Figure 2: Lion Hunt: Geneva Emotion Wheel (left) and Singapore Engagement Map.
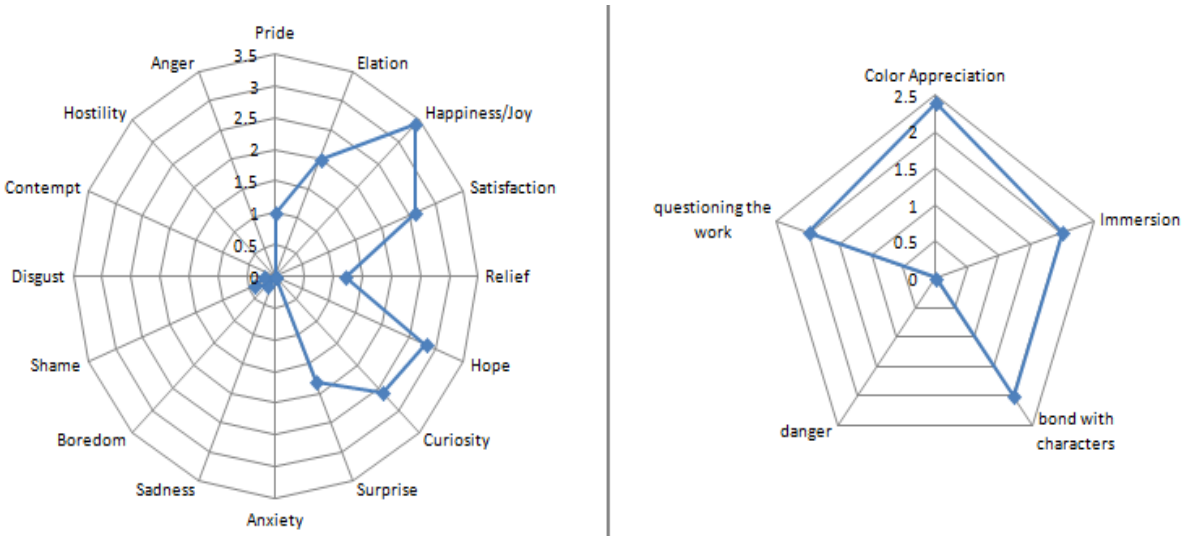

Figure 3: Yonker Ramp and His Sweetheart: Geneva Emotion Wheel (left) and Singapore Engagement Map. 

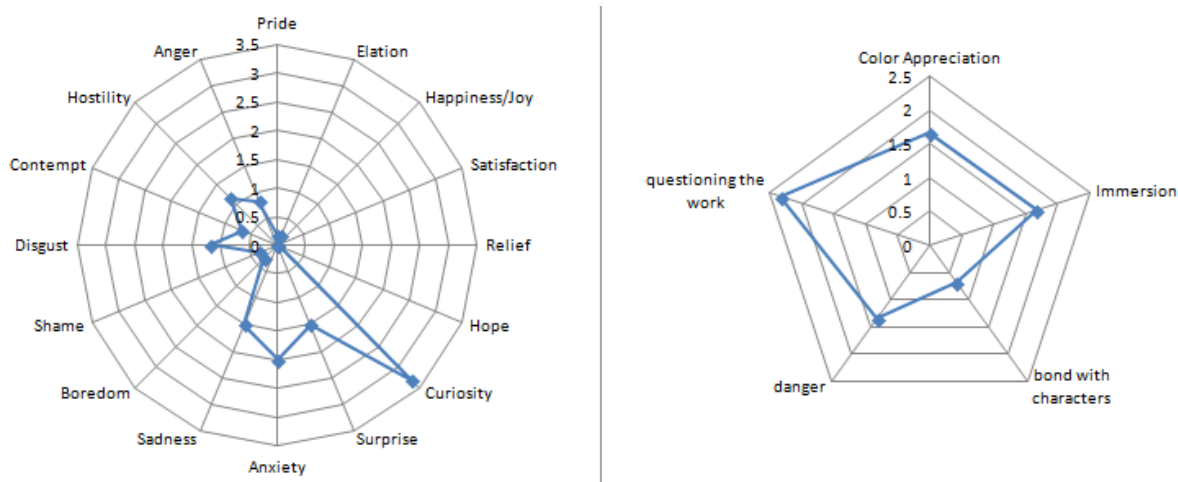

Figure 4: The Nightmare: Geneva Emotion Wheel (left) and Singapore Engagement Map.
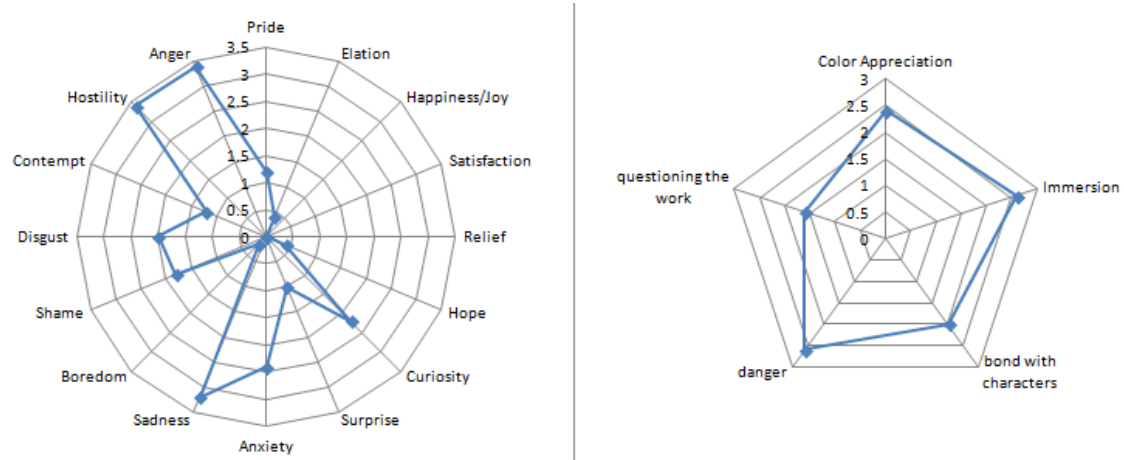

Figure 5: The Third of May 1808: Geneva Emotion Wheel (left) and Singapore Engagement Map.
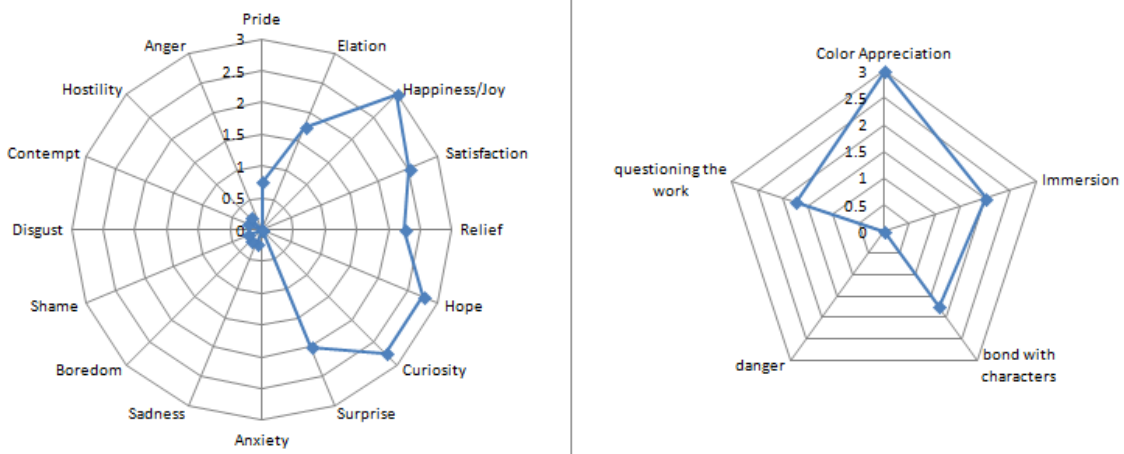

Figure 6: Garden at Sainte-Addresse: Geneva Emotion Wheel (left) and Singapore Engagement Map.
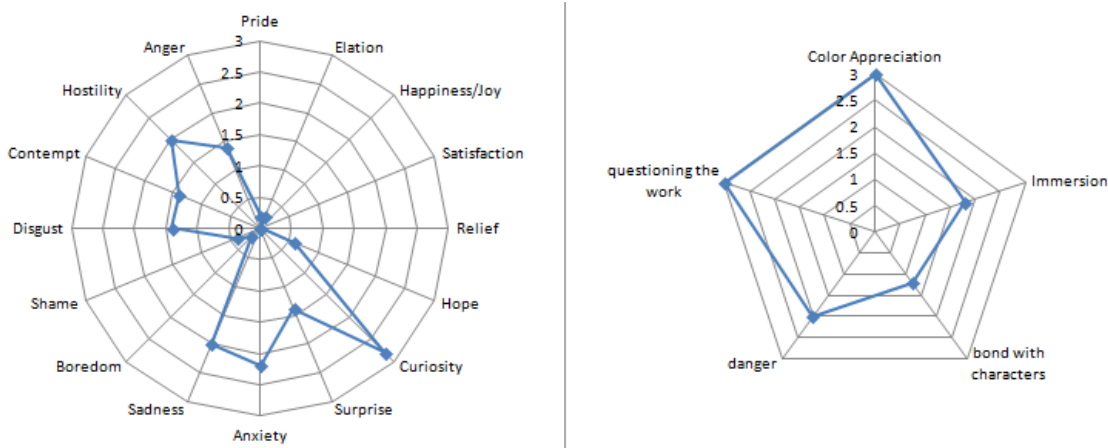

Figure 7: The Scream: Geneva Emotion Wheel (left) and Singapore Engagement Map. 

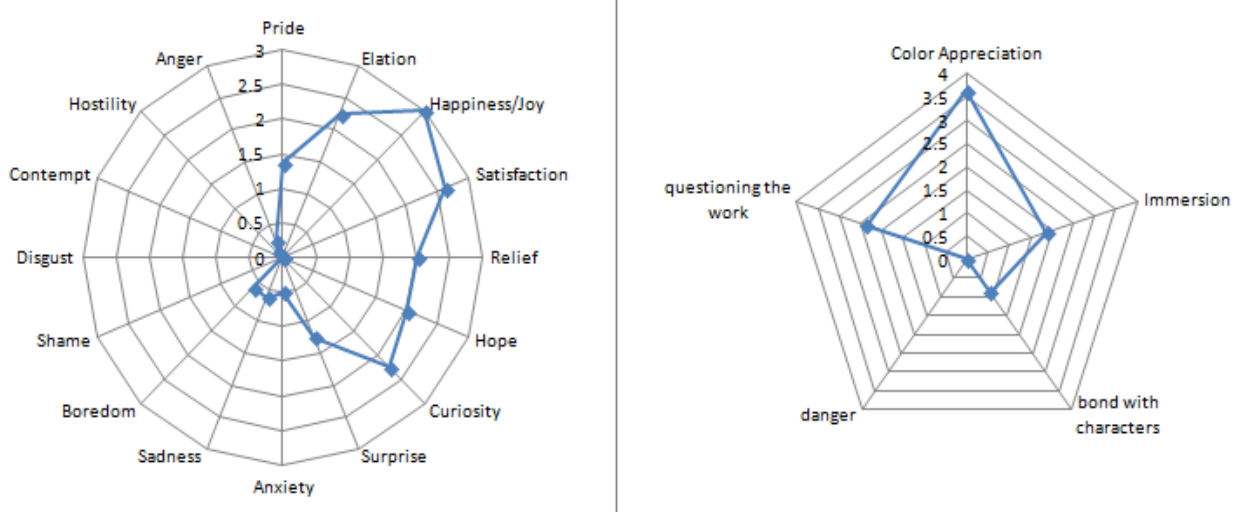

Figure 8: Flower: Geneva Emotion Wheel (left) and Singapore Engagement Map.
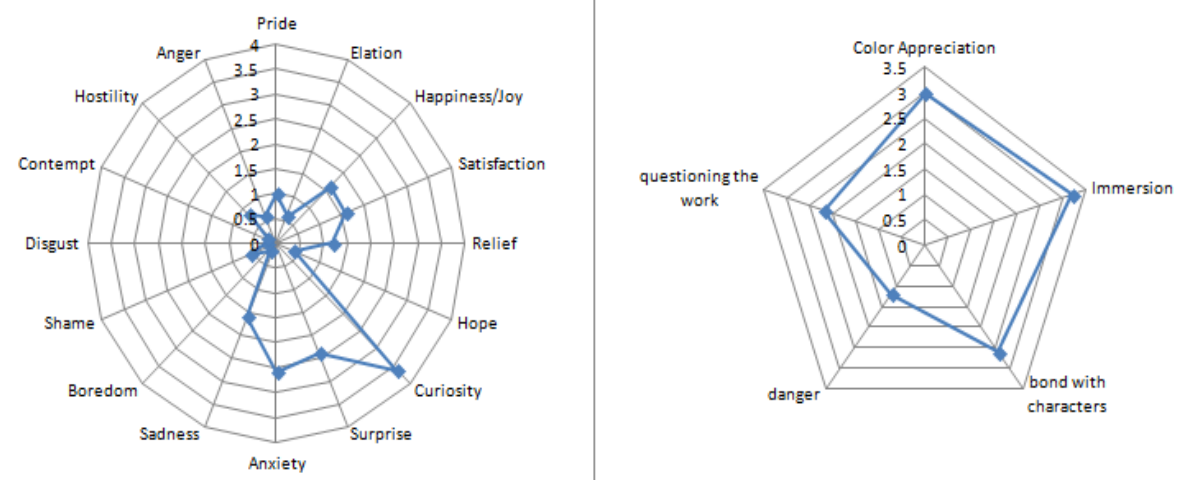

Figure 9: Heavy Rain: Geneva Emotion Wheel (left) and Singapore Engagement Map.
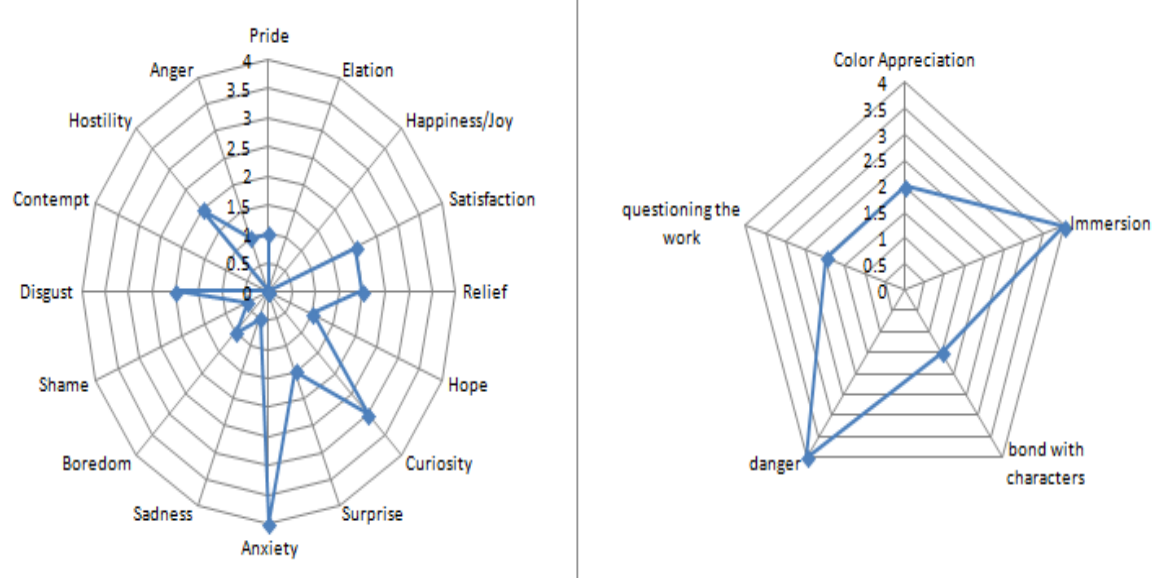

Figure 10: Amnesia: Geneva Emotion Wheel (left) and Singapore Engagement Map.
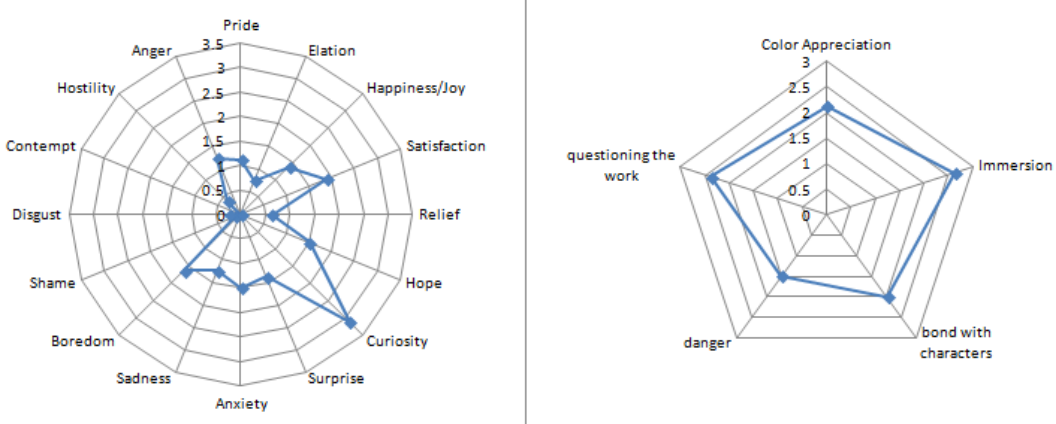
Figure 11: Ico: Geneva Emotion Wheel (left) and Singapore Engagement Map.
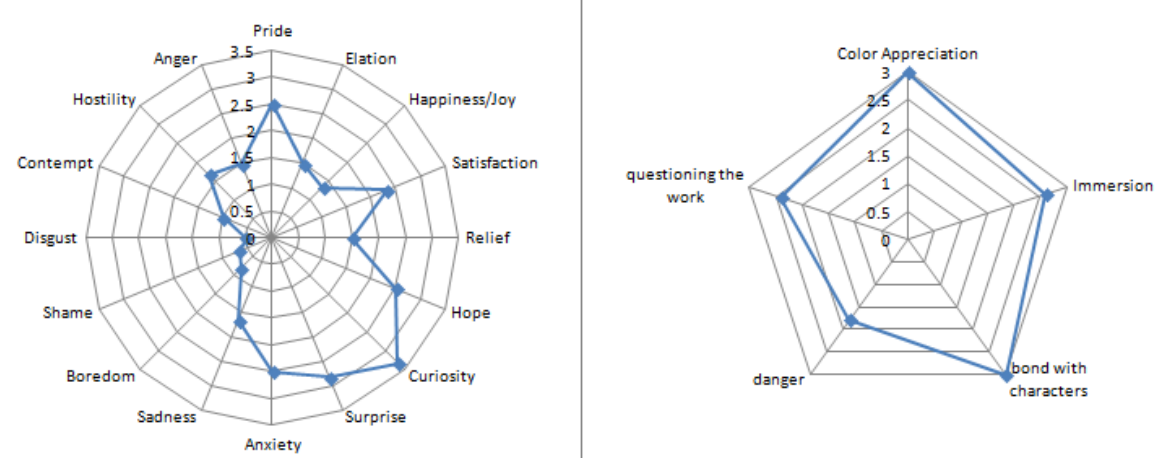

Figure 12: Shadow of the Colossus: Geneva Emotion Wheel (left) and Singapore Engagement Map.
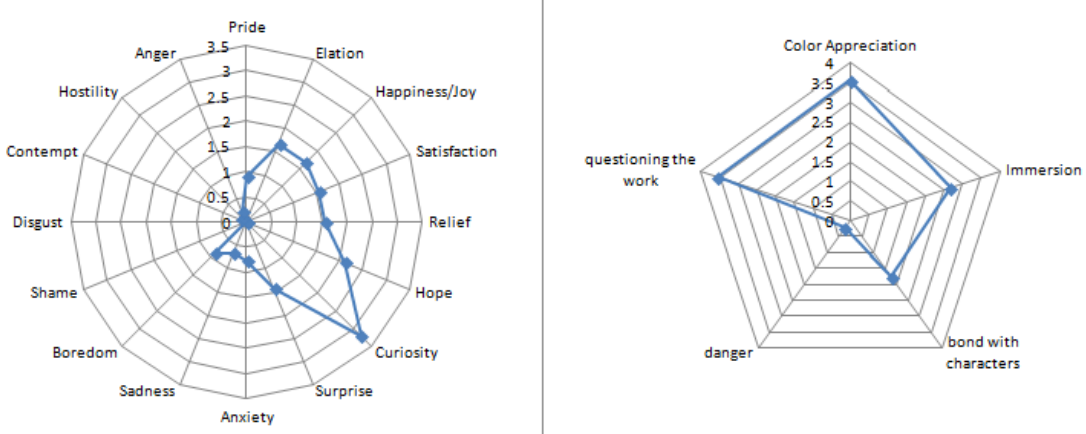

Figure 13: Journey: Geneva Emotion Wheel (left) and Singapore Engagement Map.
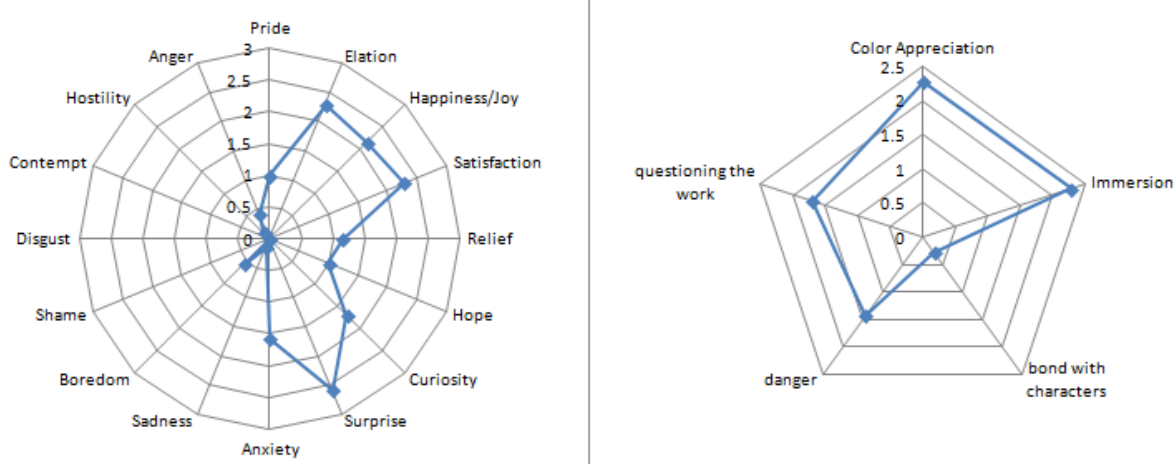

Figure 14: Child of Eden: Geneva Emotion Wheel (left) and Singapore Engagement Map.

While several of the diagrams from the two sets do look similar, for example thatgamecompany's Flower (figures 15 and 16) and Monet's Garden at Sainte-Addresse (figure 11 and 12) look remarkably close in how they engage people emotionally, we need to study whether the two groups actually showcase statistically meaningful differences or not. To accomplish this, a two-factor ANOVA $(\alpha=0.05)$ was performed on the data set, one factor being the different emotions and the other being the specific group, i.e. Artwork or Video Game. Results are shown in Table 1 for the Geneva Emotion Wheel and in Table 2 for the Singapore Engagement Map.

\begin{tabular}{|c|c|c|c|c|c|}
\hline $\begin{array}{c}\text { Source of } \\
\text { Variation }\end{array}$ & $D f$ & $M S$ & $F$ & $P$-Value & $F$ crit \\
\hline $\begin{array}{c}\text { 1st Factor } \\
\text { (Emotions) }\end{array}$ & 15 & 5.21303 & 7.79555 & $1.58 \mathrm{E}-13$ & 1.718756 \\
\hline $\begin{array}{c}\text { 2nd Factor } \\
\text { (VideoGame } \\
\text { or Art) }\end{array}$ & 1 & 1.09684 & 1.640215 & 0.201841 & 3.890348 \\
\hline
\end{tabular}

Table 1: Two Factor ANOVA $(\alpha=0.05)$ for data based on the Geneva Emotion Wheel 


\begin{tabular}{|c|c|c|c|c|c|}
\hline $\begin{array}{c}\text { Source of } \\
\text { Variation }\end{array}$ & $d f$ & $M S$ & $F$ & $P$-Value & $F$ crit \\
\hline $\begin{array}{c}\text { 1st Factor } \\
\text { (Emotions) }\end{array}$ & 4 & 4.98310 & 8.33368 & $2.05 \mathrm{E}-05$ & 2.525215 \\
\hline $\begin{array}{c}\text { 2nd Factor } \\
\text { (VideoGame } \\
\text { or Art) }\end{array}$ & 1 & 2.16644 & 3.62314 & 0.061781 & 4.001191 \\
\hline
\end{tabular}

Table 2: Two Factor ANOVA $(\alpha=0.05)$ for data based on the Singapore Engagement Map

\section{CONCLUSIONS AND FUTURE WORK}

As we can see from Tables 1 and 2, we can easily reject the null hypothesis for the first factor (different emotions), meaning that the various games and art masterpieces under study were able to actually engage people in different, unique ways, but we can't do so for the second factor, i.e. the work under analysis is a piece of art or a video game. In this case the ANOVA shows an F value well below the F critical value, meaning that we can't reject the null hypothesis in this case and we have to assume there are no statistically meaningful differences between the two groups. In other words, if we look at Art as a medium to express emotions and engage people emotionally, video games succeed in this regard exactly like well known art masterpieces and should, henceforth, be considered as an art form as well.

It can also be noted that the $F$ value for the Singapore Engagement Map, while still lower than the critical value, is relatively closer to it compared with the $F$ value for the Geneva Emotion Wheel. This is not surprising, though, since the former was devised to emphasize the interactive qualities unique to the video game medium while the second is much more general in scope.

Still, it is very interesting to realize how paintings like Goya's the Third of May 1808, for example, can manage to give a high sense of immersion and even provoke a feeling of danger in the viewers, in a way not dissimilar to video games where players can interact directly with the virtual world and not simply witness a scene. Given the positive results produced by this experiment, it may be worth expanding this approach further to keep investigating how video games engage players emotionally. For example, it may be possible to discriminate games across different genres according to specific, more relevant emotions and then understand what makes them appealing to a particular target audience.

Such analysis could also be useful as a mean to test the quality of a game, for example by looking for possible correlations between strength and variety of emotions perceived by players and critics' review scores.

\section{ACKNOWLEDGEMENTS}

This research was funded by JCU internal grant n. JCUS003/2013/RD

\section{REFERENCES}

[1] J. Kroll, 'Emotion engine'? I don't think so", Newsweek, 6/3/2000.

[2] R. Ebert, Video games can never be art, Chicago Sun-Times Blogs, 16/4/2010

[3] K. Santiago, An Argument for Game Artistry, TEDxUSC, Available online at http://bit.ly/lyxN6ra,2009.

[4] J.P. Gee, Why Game Studies Now? Video Games: a New Art Form, Games and Culture, Vol.1 No.1, pp. 58-61, 2006.

[5] A. Smuts, Are Videogames Art?, Contemporary Aesthetics, Vol.3, 2005.

[6] T.C. Devine, Games as a New Predicate for Art: What Can Arthur Danto's Theory Reveal About the Role of Games as Art, International Journal of Arts and Humanities, Vol.1(4), December 2015

[7] T.C. Devine, W.A. Presnell, S. Miller, Games as Art and Kant's Moral Dilemma: What can Ethical Theory Reveal about the Role of the Game Designer as Artist, Games and Culture, Vol.9(4), 2014

[8] C. Crawford, The Art of Computer Game Design, (Berkeley, Ca., McGraw-Hill/Osborne Media, 1984).

[9] K.R. Scherer, What are emotions? nd how can they be measured?, Social science information 44, no. 4, pp 695-729, 2005.

[10] K.R Scherer, V. Shuman, J. Fontaine and C. Soriano, The GRID meets the Wheel: Assessing emotional feeling via self-report, In Johnny R.J. Fontaine, Klaus R. Scherer \& C. Soriano (Eds.), "Components of Emotional Meaning: A sourcebook", pp. 281-298,. Oxford: Oxford University Press, 2013.

[11] R. Dillon, On the way to fun: an emotion based approach to successful game design, (Natick, MA, AKPeters, 2010).

[12] R.Dillon,,The 6-11 framework: a new approach to video game analysis and design, Proceedings GameOn Asia, Eurosis, Singapore, 2011 